\title{
Evolution of field dwarf galaxies with GEMS
}

\author{
Fabio D. Barazza ${ }^{1} \dagger$ and Shardha Jogee ${ }^{2}$ \\ ${ }^{1}$ Space Telescope Science Institute, 3700 San Martin Drive, Baltimore, MD 21218, USA \\ email: barazza@astro.as.utexas.edu \\ ${ }^{2}$ Department of Astronomy, University of Texas at Austin, RLM 15.308, Austin, TX 78712, \\ USA
}

\begin{abstract}
We present a study of the evolution of late-type field dwarfs over the last 1.5 Gyr, based on HST ACS observations carried out as part of the GEMS survey. This study is amongst the first to probe the evolution of dwarfs over such a large timescale. The comparison of structural properties, particularly size and scale length, indicates that the dwarfs in the redshift range $z \sim$ 0.01 to 0.15 (look-back times up to $1.8 \mathrm{Gyr}$ ) are more extended than local dwarfs. We argue that this difference is due to the star formation activity becoming more centrally concentrated in late-type dwarfs over the last $\sim 1.5 \mathrm{Gyr}$. We discuss several possible causes for this evolution. We also find a lack of blue compact dwarfs in the GEMS sample and interpret this as indication of the fact that strong, centrally concentrated star formation is a feature of evolved dwarfs that are entering their final stages of evolution.
\end{abstract}

Keywords. galaxies: evolution, galaxies: dwarf, galaxies: structure

\section{Introduction}

The study of dwarf galaxies outside of galaxy clusters has, so far, mainly been restricted to the Local Group and the local volume $(<10 \mathrm{Mpc})$. In these regimes the dwarf population is dominated by late-type dwarfs (henceforth referred to as dwarf irregulars, dIs), many of which are isolated, i.e. they are not associated with a giant galaxy. On the other hand, early-type dwarfs (henceforth referred to as dwarf spheroidals, dSphs) are almost absent outside of groups, but they are frequently satellites of giants and therefore quite numerous in groups. Except this morphology-density relation no structural differences have been found between group and field dwarfs. It is therefore still unclear, for instance, whether dIs are the progenitors of dSphs and what processes might lead to their transformation. Detailed studies of field dIs indicate that they generally have an older stellar population and that they would be able to produce stars for another Hubble time, if they would maintain their current low star formation rates (van Zee 2001, Grebel 2004). Hence, if no environmental interaction occurs, these objects will not end up as dSphs by passive evolution.

However, the samples of dwarf galaxies studied so far are, in general, still too small in order to draw firm conclusions. It is therefore desirable to enlarge the database not only at $z=0$, which might be realized with the Sloan Digital Sky Survey, but also for earlier epochs, where signatures of a possible evolution might be found. In this paper, we present first results based on a study of dwarfs at intermediate redshifts $(z \sim 0.01-$ 0.15 , corresponding to a look-back time of $\sim 1.8 \mathrm{Gyr}) \ddagger$, observed as part of the Galaxy Evolution from Morphology and SEDs (GEMS, Rix et al. (2004)) survey. GEMS consists

$\dagger$ Present address: Department of Astronomy, University of Texas at Austin, RLM 15.308, Austin, TX 78712, USA

$\ddagger$ We assume in this paper a flat cosmology with $\Omega_{M}=1-\Omega_{\Lambda}=0.3$ and $H_{0}=$ $65 \mathrm{~km} \mathrm{~s}^{-1} \mathrm{Mpc}^{-1}$. 

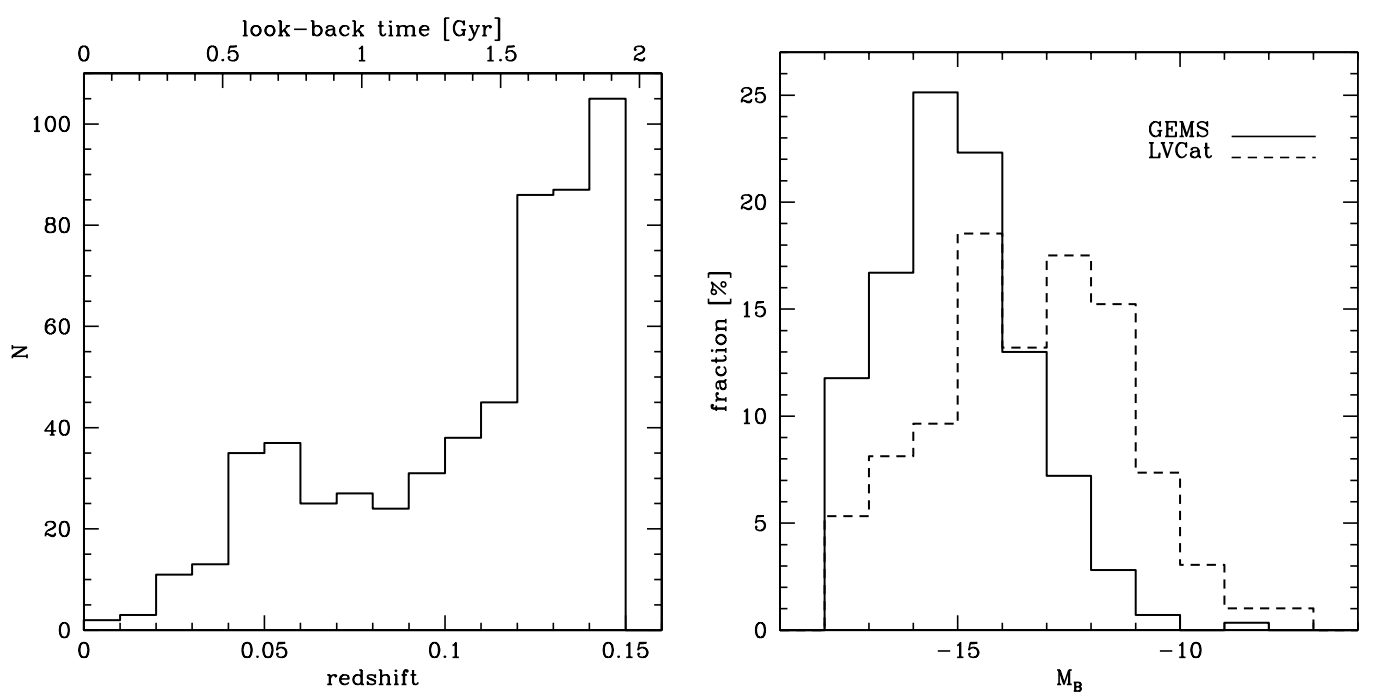

Figure 1. left: Redshift distribution of the final GEMS dwarf sample. right: Luminosity distributions of the GEMS sample and the catalog of neighboring galaxies (LVCat).

of the largest-area multi-filter survey carried out to date with the Advances Camera for Surveys (ACS) on the HubbleSpaceTelescope (HST), and might, therefore, offer the best premise for the study of dwarfs at higher redshifts. The GEMS survey produced high-resolution ( $0 . \prime 05$ correspond to $168 \mathrm{pc}$ ) images for $\sim 8300$ galaxies out to $z \sim 1.2$, for which accurate redshifts $\left(\delta_{z} /(1+z) \sim 0.02\right.$ down to $\left.R_{V \text { ega }}=24\right)$ are available from the COMBO-17 project (Wolf et al. 2004).

\section{The GEMS sample and the local comparison samples}

The largest local dwarf sample is the catalog of neighboring galaxies (Karachentsev et al. 2004), which exhibits a peak around $M_{B} \sim-14$ mag in the luminosity distribution of galaxies. We use this catalog for the comparison of the luminosity distribution of the GEMS sample. However, since this catalog does not provide additional structural parameters, we compiled a second sample of local galaxies from the literature, using the following three sources: Parodi, Barazza, \& Binggeli (2002, and references therein), a sample of 80 field and group late-type dwarfs (LV); van Zee (2000), a sample of 43 isolated dIs (ISO); Cairós et al. (2001) a sample of 15 blue compact dwarfs (BCD). These samples have been selected more or less randomly, i.e. they are not magnitude limited. But together they comprise all classes of local dwarfs commonly studied and they cover all environments outside of clusters. and are, therefore, appropriate to compare to the GEMS sample.

In order to be complete for galaxies with $M_{B}<-14 \mathrm{mag}$, we had to restrict the GEMS sample to $z<0.15$. This limit was determined by considering the detection limit of the COMBO-17 survey, i.e. at a redshift of $z \sim 0.15$ the faintest galaxies observed have $M_{B} \sim-14$ mag. (Note that we did not apply a K-correction.) For all galaxies we determined the surface brightness profile and fitted an exponential model to it. Based on a rough visual inspection we excluded all objects with signs of interaction or overlapping with other objects. The left panel of Figure 1 shows the redshift distribution of the dwarf sample. More than $70 \%$ of the objects are at look-back times of $>1$ Gyr. The right panel of Figure 1 shows the luminosity distributions of the GEMS sample and the catalog of 

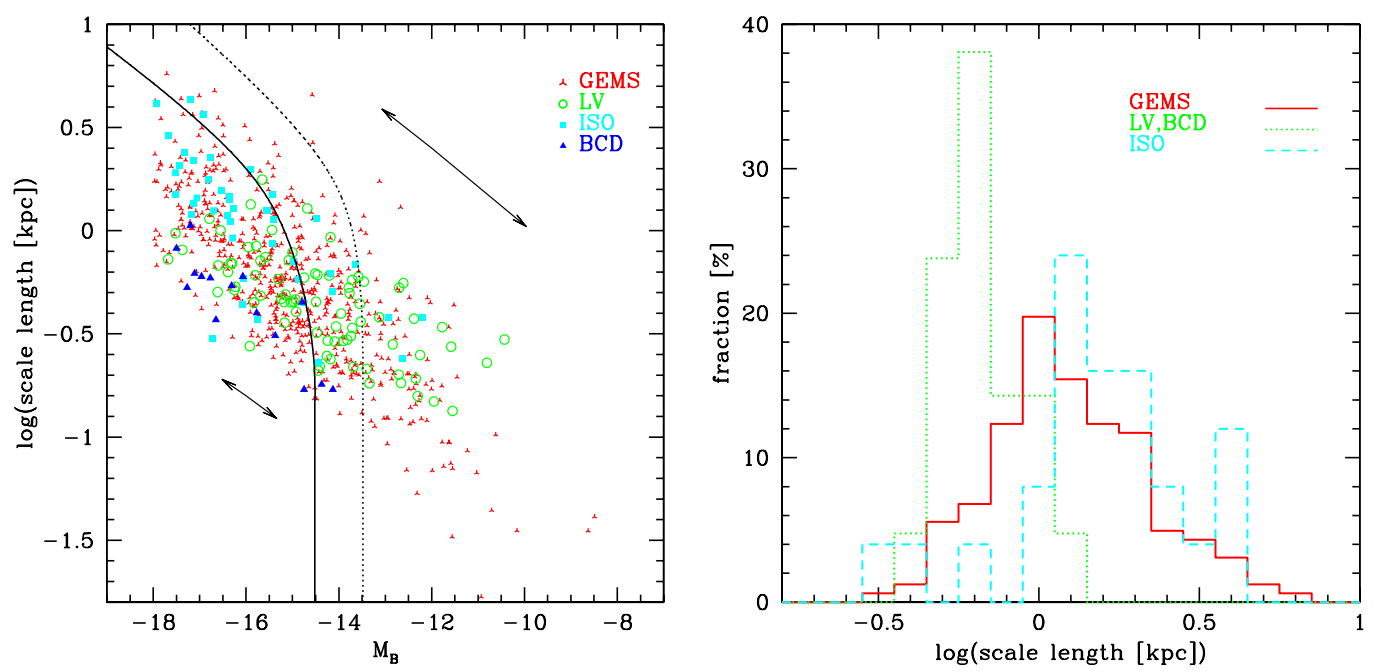

Figure 2. left: Exponential scale length versus the absolute $B$-band magnitude. The solid and dashed lines are the $100 \%$ and $50 \%$ completeness limits, respectively, of the GEMS sample. The bold line with arrows represent the typical error bars caused by redshift errors and show the direction along which such errors would move the data points. Note that the measurements for the GEMS dwarfs have been performed on the $V$-band image, which corresponds to a rest-frame band somewhere between $B$ and $V$ depending on the redshift. right: Histograms of the scale lengths for objects with $M_{B}<-16$ mag.

neighboring galaxies. The agreement is quite good (keeping in mind that the GEMS sample is roughly complete for objects with $M_{B}<-14 \mathrm{mag}$ ), which means that out study targets systems of similar luminosity across the GEMS and local samples.

\section{Results}

We compared structural parameters for the local and GEMS dwarfs. Figure 2 shows the most striking result. The GEMS dwarfs with $M_{B}<-16$ mag in the redshift range 0.01 to 0.15 seem to be more extended than the local dwarfs, particularly than LV and BCD dwarfs, which tend to be located in galaxy groups or are even satellites of giant galaxies. In contrast, no significant difference is seen between GEMS dwarfs and the ISO sample of more isolated local dwarfs. Since our study uses filter bands mainly tracing the younger stellar populations, we argue that the difference in scale lengths is the result of differences in the spatial extent of star formation sites. In GEMS dwarfs the regions of active star formation are less centrally concentrated than in the local dwarfs.

It remains to be determined whether the different extent of star formation activity in the two samples is caused by differences in environment or is simply a temporal sequence. In the latter scenario, dwarfs at earlier epochs (as typified by the GEMS dwarfs) might evolve with time into local-type dwarfs, where the extended stellar population ages passively, while star formation is sustained in the central regions. The replenishment of the central gas supply could be caused by spontaneously or tidally induced nonaxisymmetric features, such as bars or through accretion of satellites. We note that the irregular appearance of dIs does not make such an alternative unlikely and that a majority of late-type disk galaxies have bars. Moreover, this interpretation receives support from the fact that a majority of local dwarfs exhibit red color gradients, i.e. they become redder with increasing radius. In addition, in all Local Group dwarfs, for which this information 
is known, the older stellar population is more extended than the younger one (van den Bergh 1999). It is rather unlikely that these old stars have been formed near the centers of the galaxies and have subsequently been dispersed to larger radii, since the required mass losses (Ferrara \& Tolstoy 2000) would have to be caused by strong star bursts, which are not observed in the star formation histories of typical dwarfs. Thus, if they have been formed in situ, the star formation activity must have been more extended in these dwarfs in the past.

However, the better agreement between the GEMS sample and the ISO sample provides support for the scenario, in which the finding is caused by differences in the environment. This might, moreover, suggest that small galaxy groups, which are common in the local volume, only formed in the last 1-2 Gyr.

Another interesting result is the apparent lack of BCDs in the GEMS sample. This finding implicates that it is very unlikely that BCDs represent the initial stages of the evolution of late-type dwarfs or that late-type dwarfs frequently undergo a phase of strong, centrally concentrated star formation. We rather suggest that BCD-like structures are a feature of evolved dwarfs, entering their final stages of evolution.

However, it has to be kept in mind that the local samples are incomplete and that, therefore, systematics in the GEMS or local samples can affect the result. In a future paper (Barazza et al. 2005, in prep.) we will therefore compare our GEMS sample also to a sample of local dwarfs drawn from the SDSS.

\section{Acknowledgements}

F.D.B. and S.J. acknowledge support from the National Aeronautics and Space Administration (NASA) under LTSA Grant NAG5-13063 issued through the Office of Space Science. Support for GEMS was provided by NASA through GO-9500 from the Space Telescope Science Institute, STScI, which is operated by the Association of Universities for Research in Astronomy, Inc. AURA, Inc., for NASA, under NAS5-26555.

\section{References}

Barazza, F.D. et al. 2005, in prep.

Cairós, L.M., Caon, N., Vílchez, J.M., González-Pérez, J.N. \& Muñoz-Tuñón, C. 2001, ApJS 136,393

Ferrara, A. \& Tolstoy, E. 2000, MNRAS 313, 291

Grebel, E.K. 2004, in Origin and Evolution of the Elements, Carnegie Observatories Astrophysics Series, Cambridge University Press, 237

Karachentsev, I.D., Karachentseva, V.E., Huchtmeier, W.K. \& Makarov, D.I. 2004, AJ 127, 2031

Parodi, B.R., Barazza, F.D. \& Binggeli, B. 2002, A\& $A$ 388, 29

Rix, H. et al. 2004, ApJS 152, 163

van den Bergh, S. 1999, A\&SAR, 9, 273

van Zee, L 2000, $A J$ 119, 2757

van Zee, L. 2001, $A J$ 121, 2003

Wolf, C., et al. 2004, A\& $A$ 421, 913

\section{Discussion}

ToLSTOY: The very faint galaxies in your GEMS sample, being of comparable luminosity to local dSphs, do they look like them?

BARAzzA: No. They appear very compact, are forming stars, have a high surface brightness, and are also very blue. 